\title{
Left atrial geometry in an ovine ischemic mitral regurgitation model: implications for transcatheter mitral valve replacement devices with a left atrial anchoring mechanism
}

\author{
Akito Imai ${ }^{1,2+}$, Arash Khamooshian ${ }^{3 \dagger}$, Keitaro Okamoto ${ }^{1,4}$, Yoshiaki Saito ${ }^{1,5}$, Inez J. Wijdh-den Hamer ${ }^{2}$, \\ Massimo A. Mariani ${ }^{2}$, Matthew J. Gillespie ${ }^{1,6}$, Robert C. Gorman 1,7, Joseph H. Gorman III,8 and \\ Wobbe Bouma ${ }^{1,3^{*}}$
}

\begin{abstract}
Background: Transcatheter mitral valve replacement (TMVR) is a challenging, but promising minimally invasive treatment option for patients with mitral valve disease. Depending on the anchoring mechanism, complications such as mitral leaflet or chordal disruption, aortic valve disruption or left ventricular outflow tract obstruction may occur. Supra-annular devices only anchor at the left atrial (LA) level with a low risk of these complications. For development of transcatheter valves based on LA anchoring, animal feasibility studies are required. In this study we sought to describe LA systolic and diastolic geometry in an ovine ischemic mitral regurgitation (IMR) model using magnetic resonance imaging (MRI) and echocardiography in order to facilitate future research focusing on TMVR device development for (I)MR with LA anchoring mechanisms.
\end{abstract}

Methods: A group of 10 adult male Dorsett sheep underwent a left lateral thoracotomy. Posterolateral myocardial infarction was created by ligation of the left circumflex coronary artery, the obtuse marginal and diagonal branches. MRI and echocardiography were performed at baseline and 8 weeks after myocardial infarction (MI).

Results: Six animals survived to 8 weeks follow-up. All animals had grade $2+$ or higher IMR 8 weeks post-MI. All LA geometric parameters did not change significantly 8 weeks post-MI compared to baseline. Diastolic and systolic interpapillary muscle distance increased significantly 8 weeks post-MI.

Conclusions: Systolic and diastolic LA geometry do not change significantly in the presence of grade $2+$ or higher IMR 8 weeks post-MI. These findings help facilitate future tailored TMVR device development with LA anchoring mechanisms.

Keywords: Ischemic mitral regurgitation (IMR), Left atrial anchoring, Magnetic resonance imaging (MRI),

(Transcatheter) mitral valve replacement ((T)MVR)

\footnotetext{
*Correspondence: w.bouma@umcg.nl

${ }^{\dagger}$ Akito Imai and Arash Khamooshian have contributed equally to this work.

${ }^{3}$ Department of Cardiothoracic Surgery, University Medical Center Groningen, University of Groningen, Hanzeplein 1, P.O. Box 30001, 9700 RB Groningen, The Netherlands

Full list of author information is available at the end of the article
}

\section{Background}

Conventional mitral valve surgery (either repair or replacement) is the standard of care for patients with severe symptomatic mitral valve (MV) regurgitation or stenosis. Although a substantial portion of these patients are not eligible for surgery because of original author(s) and the source, provide a link to the Creative Commons licence, and indicate if changes were made. The images or other third party material in this article are included in the article's Creative Commons licence, unless indicated otherwise in a credit line to the material. If material is not included in the article's Creative Commons licence and your intended use is not permitted by statutory regulation or exceeds the permitted use, you will need to obtain permission directly from the copyright holder. To view a copy of this licence, visit http://creativecommons.org/licenses/by/4.0/. The Creative Commons Public Domain Dedication waiver (http://creativeco mmons.org/publicdomain/zero/1.0/) applies to the data made available in this article, unless otherwise stated in a credit line to the data. 
comorbidity and a high surgical risk [1]. Transcatheter mitral valve replacement (TMVR) and repair (TMVr) are enticing, but slowly evolving therapeutic options for patients with MV disease, in particular for those with a high surgical risk and those not eligible for an open procedure. Edge-to-edge leaflet repair with the MitraClip (Abbott, Chicago, IL) is the only TMVr system with the most experience to date. However, not all MV pathologies are suitable for this technique and residual moderate to severe mitral regurgitation (MR) has been reported in approximately $10 \%$ of the patients [2, 3]. For the treatment of severe MR, TMVR has emerged as an alternative. Although transcatheter aortic valve replacement (TAVR) is a well-established treatment option for patients with severe aortic stenosis, the experience with TMVR remains limited [4]. Some experience has been gained with the off-label use of TAVR devices for "valve-in-ring" remedial procedures for failed mitral valve repair [5-7], but the development of dedicated TMVR valves is currently still in its infancy [8-10]. The complexity of MV anatomy, its proximity to the left ventricular outflow tract (LVOT) and the hemodynamic forces a TMVR device must anchor and function under, have generated significant design challenges. Potential complications of TMVR are LVOT obstruction, device embolization, left ventricular dysfunction (e.g. chordae tendineae rupture) and death [11]. Despite these technical, anatomical, and functional constraints, substantial progress has been made over the last several years. The majority of TMVR devices currently being developed use a varying combination of annular interference fit, leaflet grasping or apical fixation as anchoring strategies. Left atrial anchoring strategies have been proposed, but less well explored.

The recently introduced supra-annular AltaValve (4C Medical Technologies, Brooklyn Park, Minnesota) is based on a LA anchoring mechanism and directly addresses MR without replacing the native mitral valve or disrupting the mitral annulus and leaflets, subvalvular apparatus, LVOT or aortic valve [12].

Though relatively unexplored the LA anchoring mechanism may prove a very efficient design option for development of TMVR devices. Further development of this anchoring strategy will require feasibility and efficacy studies in normal and ischemic MR (IMR) animal models. To carry out these studies animal prototypes will need to be created based on LA size and geometry. This information is not currently available in the literature. Therefore, we sought to describe LA systolic and diastolic geometry in both normal sheep and an ovine IMR model using magnetic resonance imaging (MRI) and echocardiography. This data will facilitate future research focusing on TMVR device development for (I)MR with LA anchoring mechanisms.

\section{Methods \\ Surgical protocol}

The study protocol was reviewed and approved by the University of Pennsylvania's Institutional Animal Care and Use Committee (IACUC) and was in compliance with the "Guide for the Care and Use of Laboratory Animals" compliance with (US National Institutes of Health Publication No. 85-23, revised 1996).

Ten adult male Dorsett sheep $(49.2+/-2.6 \mathrm{~kg})$ were sedated with intramuscular Ketamine injections (25$30 \mathrm{mg} / \mathrm{kg}$ ), intubated, and mechanically ventilated. General anesthesia was maintained on mixed Isoflurane (1.5-3.0\%) and oxygen, which was delivered by volumecontrolled ventilation (tidal volume $10-15 \mathrm{mg} / \mathrm{kg}$ ). The surface electrocardiogram and arterial blood pressure were monitored. All animals underwent a left lateral thoracotomy and partial removal of the fifth rib to allow ligation of the left circumflex artery, obtuse marginal branches and diagonal branches to create a posterolateral myocardial infarction (MI). After hemodynamic and electrophysiological stabilization, the thoracotomy was closed, and the animal was permitted to recover.

MRI and echocardiography were performed in all animals at baseline and 8 weeks post-MI. Six animals survived to 8 weeks follow-up. All six animals had grade $2+$ or higher IMR 8 weeks post-MI.

\section{MRI image acquisition}

MRI was performed with a 3.0 Tesla Siemens Tim Trio (Siemens, München, Germany). Imaging was performed with the use of a body matrix surface coil and a spine phased array coil. Imaging was acquired using both retrospective cardiac gating and breath-holding. Cardiac gating was performed by placement of a pressure catheter (Millar Instruments, Inc. Houston, TX) in the left ventricle $(\mathrm{LV})$. The imaging protocol for sizing of the left atrium, left ventricle, and valvular landmarks and structures was as follows:

Short axis TruFisp cines were acquired in two dimensions of the entire LV with the following parameters: slice thickness $-4 \mathrm{~mm}$, FOV-280 $\mathrm{mm} \times 166 \mathrm{~mm}$, inplane resolution $1.1 \mathrm{~mm} \times 1.1 \mathrm{~mm}, \mathrm{TR}-27.52 \mathrm{~ms}$, TE-1.46 ms, Flip angle-44.

Single slice GRE cines of the following views were acquired: 4 chamber, 2 chamber, vertical long-axis. These views were obtained with the following parameters: slice thickness $-4 \mathrm{~mm}, \mathrm{FOV}-300 \mathrm{~mm} \times 206 \mathrm{~mm}$, in-plane resolution $1.6 \mathrm{~mm} \times 1.6 \mathrm{~mm}$, TR $-28.4 \mathrm{~ms}$, TE- $-2.85 \mathrm{~ms}$, Flip angle-12. 
Single slice phase contrast through-plane at mitral annulus for IMR evaluation was obtained with the following parameters: slice thickness $-4 \mathrm{~mm}$, FOV$300 \mathrm{~mm} \times 206 \mathrm{~mm}$, in-plane resolution $2.1 \times 1.6$, TR $-40.85 \mathrm{~ms}$, TE-3.33 ms, Flip angle-12, VENC range $75 \mathrm{~cm} / \mathrm{s}$.

All MRI images were suitable for analysis. Reconstructed images were transferred to an external workstation and subsequently analyzed.

\section{MRI image analysis}

The following parameters were measured by MRI at baseline and 8 weeks after myocardial infarction: (1) left atrial inner diameter (LAID), (2) length of the left atrium ( $L A L)$, (3) annulus plane to LAID (AP-LAID), (4) left atrial roof length (ID-ID), (5) mitral valve tethering height (MVTH),

(6) left atrial roof to myocardium distance (LAR-MYC),

(7) LAR to papillary muscle base distance (LAR-PMB),

(8) papillary muscle to papillary muscle distance (PMRPMR) (Fig. 1).

\section{Statistics}

Continuous variables were expressed as mean \pm standard deviation (SD). Comparisons within groups were performed using the paired samples $t$-test (two-sided) as appropriate for continuous variables. All calculations were performed using SPSS Statistics (IBM SPSS Statistics 22.0; IBM Corporation, Chicago, IL). Statistically significant differences were established at $P<0.05$.

\section{Results}

\section{Geometric MRI data}

Ten animals were included in this study at baseline, 6 animals were alive 8 weeks post-MI and underwent followup MRI. The geometric MRI data are shown in Table 1. Eight weeks post-MI interpapillary muscle distance enlarged significantly both in systole and diastole. Grade $2+$ or more IMR was observed after 8 weeks in all cases. All LA geometric parameters did not change significantly 8 weeks post-MI compared to baseline.

\section{Discussion}

TMVR and TMVr are new therapeutic options for patients with mitral valve disease. Especially TMVR has emerged as an alternative to surgery for patients with an increased operative risk [2]. However, TMVR still faces many technical and anatomical challenges [11]. A variety of TMVR designs are currently under investigation. TMVR design has to take into account the proximity of the MV to the LVOT, the complexity of the MV itself and hemodynamic forces. TMVR devices should not obstruct the LVOT, fit the MV annulus well, not cause left ventricular dysfunction by disrupting the chords and be antithrombogenic. The main challenge is designing a robust anchoring mechanism which meets these standards. Different designs have been introduced, ranging from annular interference fit, leaflet grasping, apical fixation to LA anchoring strategies [13].

Our group has explored several leaflet, annular and left ventricular TMVR anchoring strategies and have

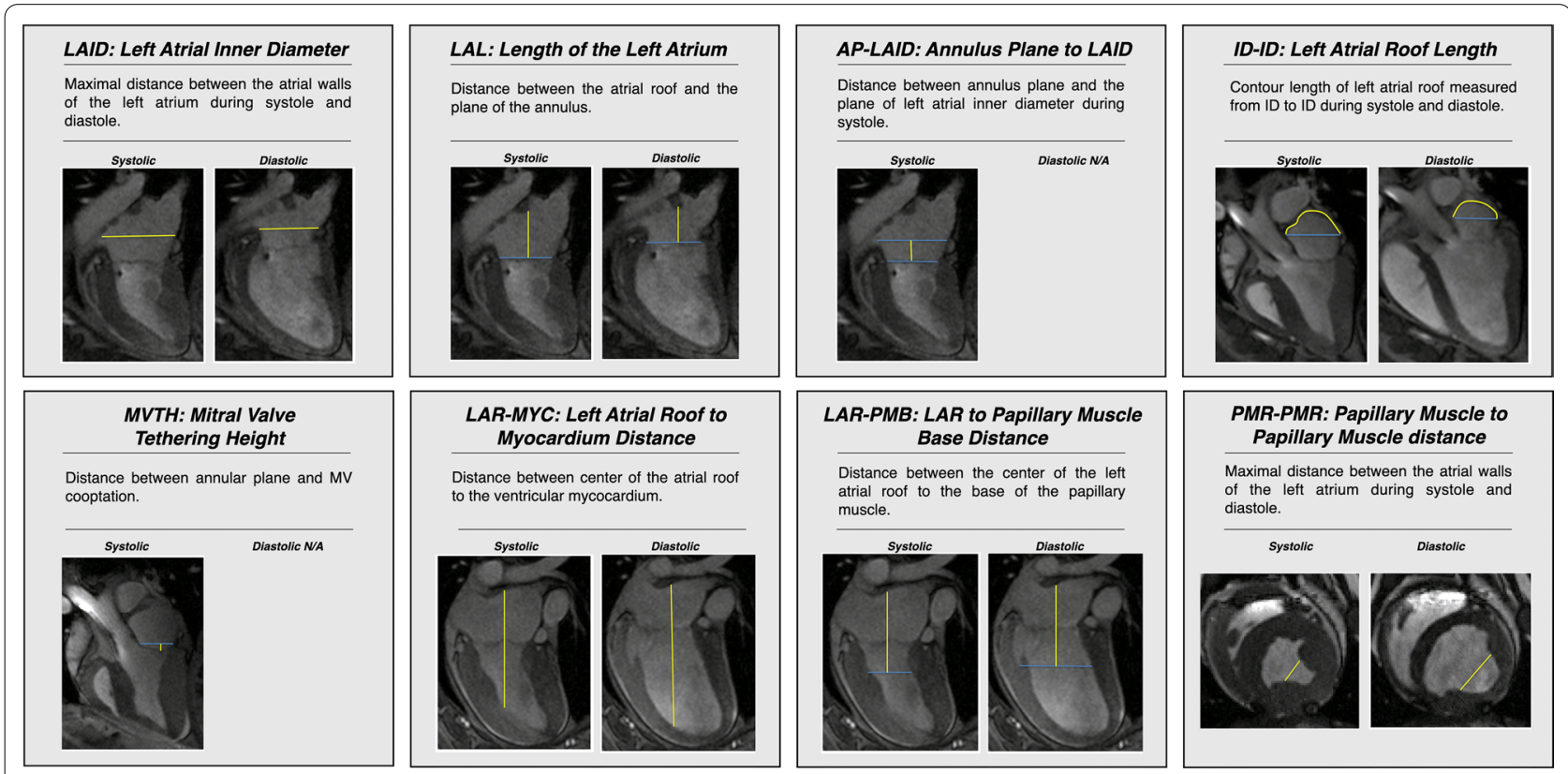

Fig. 1 MRI parameters measured at baseline and 8 weeks post myocardial infarction 
Table 1 Geometric ovine MRI data at baseline and 8 weeks post myocardial infarction (systolic and diastolic)

\begin{tabular}{|c|c|c|c|c|c|c|}
\hline \multirow[t]{2}{*}{ Variable $^{a}$} & \multicolumn{3}{|l|}{ Systolic } & \multicolumn{3}{|l|}{ Diastolic } \\
\hline & Baseline & 8 weeks post-MI & $P$ value & Baseline & 8 weeks post-MI & $P$ value \\
\hline Let atrial inner diameter (LAID), mm & $55.9 \pm 6.9$ & $53.8 \pm 4.8$ & 0.580 & $44.7 \pm 3.5$ & $47.0 \pm 6.6$ & 0.566 \\
\hline Left atrial length (LAL), mm & $33.2 \pm 4.0$ & $33.7 \pm 2.8$ & 0.847 & $26.6 \pm 3.6$ & $31.0 \pm 2.3$ & 0.091 \\
\hline Annulus plane to LAID, mm & $18.5 \pm 2.7$ & $19.5 \pm 1.9$ & 0.381 & - & - & - \\
\hline Left atrial roof contour, mm & $61.4 \pm 5.0$ & $62.2 \pm 2.3$ & 0.754 & $46.7 \pm 6.7$ & $52.8 \pm 5.6$ & 0.165 \\
\hline Mitral valve height, mm & $4.6 \pm 0.9$ & $4.6 \pm 1.0$ & 0.961 & - & - & - \\
\hline Left atrial roof to myocardium, mm & $77.7 \pm 4.5$ & $80.8 \pm 5.0$ & 0.276 & $92.1 \pm 4.2$ & $88.5 \pm 5.0$ & 0.201 \\
\hline Left atrial roof to papillary muscle base, $\mathrm{mm}$ & $55.8 \pm 4.6$ & $58.0 \pm 2.9$ & 0.423 & $49.9 \pm 5.3$ & $56.8 \pm 3.1$ & 0.057 \\
\hline Interpapillary muscle distance, mm & $16.8 \pm 4.5$ & $28.5 \pm 6.1$ & $<0.001$ & $29.3 \pm 4.8$ & $36.9 \pm 3.7$ & 0.004 \\
\hline
\end{tabular}

$\mathrm{LAID}=$ left atrial inner diameter; $\mathrm{LAL}=$ left atrial length; $\mathrm{MI}=$ myocardial infarction; $\mathrm{MRI}=$ magnetic resonance imaging

${ }^{\text {a }}$ Data are presented as mean \pm SD

found that our previously published normal and IMR ovine LV and annular data [14-16] to be extremely helpful in designing prototypes for testing in animal models. We have not previously studied ovine LA anatomy and the literature on ovine LA anatomy is very sparse. To date, only one device has been proposed with a LA anchoring mechanism; the supra-annular AltaValve (4C Medical Technologies, Brooklyn Park, $\mathrm{MN}$ ) [12]. We believe LA anchoring is a potentially effective strategy and undertook the current study to provide geometric baseline and post-infarct ovine LA data to facilitate its development.

Benefits of a LA anchoring design include the lack of need to replace the native mitral valve or disrupt the mitral annulus and leaflets, subvalvular apparatus, LVOT and aortic valve. In this study we provide baseline and 8 weeks post-infarct data regarding ovine LA geometry. This study shows that LA geometry remains relatively stable in the presence of grade $2+$ or more IMR in the first 8 weeks post-MI. The specific implications of these findings are that initial development and testing of TMVR devices with a LA anchoring mechanism (such as a LA nitinol stent) can be done in normal sheep. This is an important finding, because it eliminates the additional time and expense required to produce the ovine IMR model. Once a design has been shown to be effective in normal sheep, subsequent testing can then be conducted more efficiently in our ovine chronic IMR models.

Device testing in long-term IMR ovine models are a next step in moving towards human testing. While this study shows that LA geometry remains relatively stable in the presence of grade $2+$ or more IMR in the first 8 weeks post-MI, additional studies are required to explore what happens after a longer timeframe in this ovine IMR model.
It should be stressed that the current data is only helpful in developing animal prototypes for proof of principle studies. Devices for human use, while being similarly designed, will have to be re-scaled based on human LA geometrical data. Our group is currently analyzing our large library of human IMR echocardiography studies to generate this information.

\section{Limitations}

The number of animals in this study is relatively small. Although we used a chronic post-MI model, additional LA geometrical and functional changes are expected to occur over a long period of time in the presence of IMR. This may have implications for LA anchoring TMVR device development and is currently being studied. IMR was measured semiquantitatively with jet area/LA area. Alternative validated methods for quantitative IMR severity assessment, including regurgitant volume and effective regurgitant orifice, were not available in this study.

\section{Conclusions}

Systolic and diastolic LA geometry do not change significantly in the presence of grade 2+or higher IMR 8 weeks post-MI in an ovine model. These findings help facilitate future tailored TMVR device development with LA anchoring mechanisms.

\footnotetext{
Abbreviations

(AP-)LAID: (Annulus plane to) left atrial inner diameter; IACUC: Institutional animal care and use committee; ID-ID: Left atrial roof length; (I)MR: (Ischemic) mitral regurgitation; $L A(L)$ : Left atrial (length); LAR-MYC: Left atrial roof to myocardium distance; LAR-PMB: Left atrial roof to papillary muscle base; LV: Left ventricle; LVOT: Left ventricular outflow tract; MI: Myocardial infarction; MRI: Magnetic resonance imaging; $\mathrm{MV}(\mathrm{TH})$ : Mitral valve (tethering height);
} 
PMR-PMR: Papillary muscle to papillary muscle distance; SD: Standard deviation; TAVR: Transcatheter aortic valve replacement; (T)MVr: (Transcatheter) mitral valve repair; (T)MVR: (Transcatheter) mitral valve replacement.

\section{Acknowledgements}

None.

\section{Authors' contributions}

All authors meet ICMJE guidelines for contribution. Al, AK, KO, and YS collected the data and wrote the manuscript. $\mathrm{Al}, \mathrm{AK}, \mathrm{KO}, \mathrm{YS}, \mathrm{JG}$, and WB interpreted statistics. IW, MM, MG, RG, JH and WB participated in the design of the manuscript and they revised and critically reviewed the manuscript. All authors have read, critically reviewed, and approved the final manuscript.

\section{Funding}

This research project was supported by grants from the National Heart, Lung and Blood Institute of the National Institutes of Health, Bethesda, MD (HL073021, HL-147256 and HL-103723).

\section{Availability of data and materials}

The datasets used and/or analysed during the current study are available from the corresponding author on reasonable request.

\section{Declarations}

\section{Ethics approval and consent to participate}

The study protocol was reviewed and approved by the University of Pennsylvania's Institutional Animal Care and Use Committee (IACUC) and was in compliance with the "Guide for the Care and Use of Laboratory Animals" compliance with (US National Institutes of Health Publication No. 85-23, revised 1996).

\section{Consent for publication}

Not applicable.

\section{Competing interests}

The authors declare that they have no competing interests.

\section{Author details}

${ }^{1}$ Gorman Cardiovascular Research Group, University of Pennsylvania, Philadelphia, PA, USA. ${ }^{2}$ Department of Cardiovascular Surgery, University of Tsukuba, Tsukuba, Ibaraki, Japan. ${ }^{3}$ Department of Cardiothoracic Surgery, University Medical Center Groningen, University of Groningen, Hanzeplein 1, P.O. Box 30001, 9700 RB Groningen, The Netherlands. ${ }^{4}$ Department of Cardiovascular Surgery, Oita University, Oita, Japan. ${ }^{5}$ Department of Thoracic and Cardiovascular Surgery, Hirosaki University School of Medicine, Hirosaki, Japan. ${ }^{6}$ Department of Pediatrics, Children's Hospital of Philadelphia, University of Pennsylvania, Philadelphia, PA, USA. ${ }^{7}$ Department of Surgery, University of Pennsylvania, Philadelphia, PA, USA. ${ }^{8}$ Onocor LLC, Philadelphia, PA, USA.

Received: 11 March 2021 Accepted: 20 September 2021 Published online: 10 October 2021

\section{References}

1. Mirabel M, lung B, Baron G, Messika-Zeitoun D, Detaint D, Vanoverschelde $J$, et al. What are the characteristics of patients with severe, symptomatic, mitral regurgitation who are denied surgery? Eur Heart J. 2007;28:1358-65.

2. Nishimura RA, Vahanian A, Eleid MF, Mack MJ. Mitral valve disease-current management and future challenges. Lancet. 2016;387:1324-34.

3. Chiarito M, Pagnesi M, Martino EA, Pighi M, Scotti A, Biondi-Zoccai G, et al. Outcome after percutaneous edge-to-edge mitral repair for functional and degenerative mitral regurgitation: a systematic review and metaanalysis. Heart. 2018;104:306-12.

4. Wyler von Ballmoos MC, Kalra A, Reardon MJ. Complexities of transcatheter mitral valve replacement (TMVR) and why it is not transcatheter aortic valve replacement (TAVR). Ann Cardiothorac Surg. 2018;7:724-30.

5. Ye J, Cheung A, Yamashita M, Wood D, Peng D, Gao M, et al. Transcatheter aortic and mitral valve-in-valve implantation for failed surgical bioprosthetic valves: an 8-year single-center experience. JACC Cardiovasc Interv. 2015;8:1735-44.

6. Eleid MF, Whisenant BK, Cabalka AK, Williams MR, Nejjari M, Attias D, et al. Early outcomes of percutaneous transvenous transseptal transcatheter valve implantation in failed bioprosthetic mitral valves, ring annuloplasty, and severe mitral annular calcification. JACC Cardiovasc Interv. 2017:10:1932-42.

7. Yoon SH, Whisenant BK, Bleiziffer S, Delgado V, Dhoble A, Schofer N, et al. Outcomes of transcatheter mitral valve replacement for degenerated bioprostheses, failed annuloplasty rings, and mitral annular calcification. Eur Heart J. 2019:40:441-51.

8. Badhwar V, Sorajja P, Duncan A, Thourani V, Schaefer U, Grayburn P, et al. Mitral regurgitation severity predicts one-year therapeutic benefit of Tendyne transcatheter mitral valve implantation. Eurolntervention. 2019;15:e1065-71.

9. Perpetua EM, Reisman M. The Tendyne transcatheter mitral valve implantation system. Eurolntervention. 2015;11(Suppl W):W78-9.

10. Piazza N, Overtchouk P, Ben-Shoshan J. Predicting TMVR outcomes-the Tendyne experience. Eurolntervention. 2019;15:e1033-4.

11. El Hajj SC, Eleid MF. Transcatheter mitral valve replacement: an update on the current literature. Curr Treat Options Cardiovasc Med. 2019;21:35.

12. Ferreira-Neto AN, Dagenais F, Bernier M, Dumont E, Freitas-Ferraz AB, Rodes-Cabau J. Transcatheter mitral valve replacement with a new supraannular valve: first-in-human experience with the AltaValve system. JACC Cardiovasc Interv. 2019:12:208-9.

13. Del Val D, Ferreira-Neto AN, Wintzer-Wehekind J, Dagenais F, Paradis JM, Bernier $\mathrm{M}$, et al. Early experience with transcatheter mitral valve replacement: a systematic review. J Am Heart Assoc. 2019;8:e013332.

14. Gorman JH 3rd, Gorman RC, Jackson BM, Hiramatsu Y, Gikakis N, Kelley ST, et al. Distortions of the mitral valve in acute ischemic mitral regurgitation. Ann Thorac Surg. 1997;64:1026-31.

15. Gorman JH 3rd, Gorman RC, Plappert T, Jackson BM, Hiramatsu Y, StJohnSutton MG, et al. Infarct size and location determine development of mitral regurgitation in the sheep model. J Thorac Cardiovasc Surg. 1998;115:615-22.

16. Siefert AW, Jimenez JH, Koomalsingh KJ, Aguel F, West DS, Shuto T, et al. Contractile mitral annular forces are reduced with ischemic mitral regurgitation. J Thorac Cardiovasc Surg. 2013;146:422-8.

\section{Publisher's Note}

Springer Nature remains neutral with regard to jurisdictional claims in published maps and institutional affiliations. 